\title{
A INFLUENNCIA INTERPESSOAL EM REDES SOCIAIS VIRTUAIS E AS DECISÕES DE CONSUMO
}

\author{
INTERPERSONAL INFLUENCE IN VIRTUAL SOCIAL \\ NETWORKS AND CONSUMER DECISIONS
}

\author{
Recebido 15-03-2012 \\ Aceito 29-07-2012 \\ Eduardo Botti Abbade ${ }^{1}$ \\ Andiara Della Flora ${ }^{2}$ \\ Greice de Bem Noro ${ }^{3}$
}

\section{RESUMO}

Este estudo teve como objetivo analisar a postura de estudantes universitários com relação à influência interpessoal em redes sociais virtuais no processo decisório de consumo de estudantes universitários. Para isso, foi conduzido um survey com 200 estudantes universitários de uma Instituição de Ensino Superior (IES) localizada em Santa Maria, Rio Grande do Sul. A amostra foi obtida por adesão, e o instrumento de coleta de dados foi aplicado em ambiente virtual. Foram adaptadas escalas para mensurar e avaliar a propensão dos estudantes universitários para influenciar e serem influenciados pelos seus contatos de redes sociais virtuais. Os resultados sugerem que as escalas adaptadas são satisfatórias para mensurar aquilo a que elas se propõem. Também foi constatado que homens apresentam maior capacidade de influenciar as opiniões de seus contatos sociais virtuais. Já o tempo de acesso à internet influencia positivamente e significativamente a propensão dos usuários para serem influenciados por seus contatos sociais virtuais. A correlação existente entre a capacidade de influenciar e a propensão de ser influenciado é significativa e positiva.

Palavras-chave: Marketing Eletrônico; Redes Sociais Virtuais; Comunicação Boca a Boca; Comportamento do Consumidor.

1 Possui graduação em Administração de Empresas pela Universidade Federal de Santa Maria - UFSM, especialização em Estatística e Modelagem Quantitativa pela Universidade Federal de Santa Maria - UFSM, mestrado em Administração pela Universidade Federal do Paraná - UFPR. Atualmente é doutorando em Agronegócios Universidade Federal do Rio Grande - UFRGS e professor assistente do Centro Universitário Franciscano - UNIFRA. Santa Maria, Rio Grande do Sul, Brasil. E-mail: eduardo@unifra.br.

2 Possui graduação em Administração pelo Centro Universitário Franciscano -UNIFRA, especialização em Gestão Empresarial com ênfase em Marketing pela Escola Superior de Propaganda e Marketing - ESPM. Atualmente é gerente administrativa no restaurante The Park Bar e Restaurante. Santa Maria, Rio Grande do Sul, Brasil. E-mail: andizinhadf@hotmail.com.

3 Possui graduação em Administração de Empresas pela Universidade Federal de Santa Maria - UFSM, mestrado em Engenharia de Produção pela Universidade Federal de Santa Maria - UFSM. Atualmente é doutoranda em Administração pela Universidad Nacional de Misiones - 


\begin{abstract}
This study aimed to analyze the attitude of college students regarding to interpersonal influence in virtual social networks related to consume decisions. It was conducted a survey with 200 college students from an Institution of Higher Education located in Santa Maria/RS. The sample was obtained through voluntary adhesion and the data collection instrument was applied in a virtual environment. Scales were adapted to measure and evaluate the propensity of students to influence and be influenced by their virtual contacts. The results suggest that the scales adapted are satisfactory to measure what they intend to do. The study also found that men are more able to influence the opinions of their virtual social contacts. On the other hand, the time dedicated to access the Internet positively and significantly influences the propensity of users to be influenced by their virtual social contacts. The correlation between the ability to influence the propensity to be influenced is significant and positive.
\end{abstract}

Keywords: Eletronic Marketing; Virtual Social Networks; Word-of-Mouth; Consumer Behaviour.

\title{
1 INTRODUÇÃO
}

A revolução digital, estimulada pela ampliação do acesso à internet e das novas ferramentas virtuais desenvolvidas, tem alterado o comportamento dos consumidores, que passaram a utilizar os meios digitais como ferramenta importante para a busca de informações sobre produtos e empresas. Confirmando essa ideia, uma pesquisa publicada na revista HSM Management revela que $88 \%$ das pessoas entrevistadas afirmam comparar preços e características de produtos na internet antes de realizar uma compra (STANLEY, 2008).

Dessa forma, a constante evolução da internet possibilita, cada vez mais, interação e troca de informações entre consumidores, já que na Web 2.0 os elementos primordiais desse contato são a colaboração, a interatividade e a geração de conteúdo. Juntamente a isso, surgem as redes sociais virtuais, que são redes de intercâmbio social desenvolvidas na internet, em que as pessoas trocam diferentes tipos de informações (DAMBRÓS, 2007).

$\mathrm{O}$ Brasil tem apresentado um crescimento acentuado quanto ao acesso à internet. Em 2005 , cerca de $13,7 \%$ dos domicílios permanentes brasileiros tinham acesso à internet. Já em 2009 a quantidade de domicílios com acesso à rede virtual foi de $27,4 \%$ do total de domicílios. As regiões brasileiras que apresentaram maior proporção de domicílios com acesso à internet foram as regiões sudeste e sul, com $35,4 \%$ e $32,8 \%$, respectivamente (IBGE, 2012). Além disso, uma pesquisa realizada pela Jeffrey Group revelou que o acesso a redes sociais é o principal motivo para $75 \%$ dos internautas brasileiros acessarem a internet. Outra evidência importante do mesmo estudo foi a constatação de que $95,2 \%$ dos internautas acessam o Facebook, enquanto $79,2 \%$ usam o Twitter. Outras ferramentas sociais apresentaram índices de acesso menores, como MSN $(46,6 \%)$, Orkut $(38,6 \%)$, Skype $(33,6 \%)$ e Linkedln $(21,6 \%)$ (JEFFREY GROUP, 2012).

$\mathrm{O}$ fenômeno da colaboração entre consumidores por meio da internet modificou-se radicalmente com o surgimento das redes sociais virtuais, que propuseram aos usuários novas formas de interagir, expressar-se e opinar sobre os mais diversos assuntos, inclusive produtos. O que mudou nos últimos anos é que as pessoas começaram a contribuir ativamente com seus dados, compartilhando as situações que vivenciam, seus interesses e seus relacionamentos. As comunidades digitais funcionam como um repositório de opiniões e experiências, pois a facilidade de uso das ferramentas de comunicação on-line, aliada à vontade de expressar-se, leva as pessoas a falarem sobre qualquer assunto na rede: atividades pessoais, política nacional ou, ainda, produtos e marcas, geralmente refletindo suas experiências com relação a esses.

Dessa forma, muitos consumidores estão baseando suas decisões de compra nas informações, nas opiniões e nos relatos sobre experiências descritas nas redes sociais. Um estudo que 
abrangeu um total de 4,6 mil pessoas entrevistadas no Brasil, no Oriente Médio, na Alemanha, no Reino Unido e nos Estados Unidos revelou que $56 \%$ dos brasileiros entrevistados admitiram seguir sugestões de conhecidos da rede. Outro dado revelador afirma que $40 \%$ dos entrevistados brasileiros estão dispostos a aceitar recomendações feitas por desconhecidos, o que evidencia o grau de confiança absoluto que os internautas depositam na rede (SEGALLA, RIBEIRO e BARIFOUSE, 2007).

A crescente participação do Brasil em estatísticas relacionadas à internet e às redes sociais virtuais demonstra a importância que ambas adquiriram no dia a dia das pessoas, sendo percebidas pelos consumidores como fontes que fornecem informações verídicas, mais acessíveis e mais rápidas do que os outros meios. Assim, o objetivo geral do presente estudo foi analisar a propensão a ser influenciado e a capacidade de influenciar de estudantes universitários em redes sociais virtuais. Além disso, o estudo objetivou avaliar o impacto do gênero, do tempo de uso de internet, da idade e da renda familiar dos estudantes pesquisados nos seus comportamentos de propensão de influência e de capacidade de influenciar em redes sociais virtuais.

As redes sociais virtuais, que inicialmente surgiram como um meio de fomentar a interação e o relacionamento entre pessoas, hoje são utilizadas como fonte de informação à medida que as pessoas passam a expor suas impressões acerca de produtos e serviços. Como redes sociais virtuais pode-se citar Blogs, Facebook, Fóruns de Discussão, Orkut, Twitter, Linkedln, entre outras. Essas redes possuem ferramentas que facilitam a comunicação e a exposição da opinião pessoal, incluindo Posts sobre determinado assunto, juntamente com os respectivos comentários dos leitores, comunidades específicas em que o usuário pode dar a sua opinião, divulgação de links interessantes e a recomendação feita em meio digital de certos produtos que o usuário envia a sua lista de contatos.

A escolha desse tema ocorreu tendo em vista que a internet e as redes sociais estão cada vez mais inseridas no cotidiano dos consumidores, despertando a necessidade de pesquisar o grau de influência desses fatores nas escolhas feitas pelo público consumidor estudantil. Dessa forma, esta pesquisa é importante, pois identificou o comportamento dos consumidores locais com relação à busca de informações acerca de produtos e serviços. Outro fator relevante é que, ao identificar uma nova tendência, a presente pesquisa contribui com empresas, que precisam se adaptar a essa nova realidade virtual e, também, monitorar o que é dito sobre seus produtos e serviços na internet.

\section{COMUNICAÇÃO BOCA A BOCA (WORD-OF-MOUTH) E BOCA A BOCA ELETRÔNICA (E-WOM)}

O termo "boca a boca" (Word-of-Mouth) foi inicialmente utilizado por William Whyte, em 1954, em um artigo publicado pela revista Fortune, que o relacionava com a capacidade de influência interpessoal para a disseminação de informações a respeito de produtos e serviços entre consumidores (KIMMEL, 2003). O conceito de Word-of-Mouth já é amplamente conhecido e utilizado tanto no meio prático como no meio acadêmico. Sua concepção de troca de informações entre consumidores por meios informais ganhou novos patamares de importância com o advento da internet e das redes sociais virtuais. O que antes era considerado um fenômeno relativamente isolado e delimitado por aspectos geográficos e de conectividade interpessoal limitada atualmente ganha proporções de escopo global e sem fronteiras.

A comunicação boca a boca, compreendida como a comunicação informal existente entre consumidores reais e/ou potenciais relacionada a produtos, serviços, marcas ou empresas, propaga-se de forma intensa e depende do tipo de relação que o disseminador da informação 
(emissor) tem com o receptor. $\mathrm{O}$ laço informal da amizade ou o conhecimento prévio existente entre as partes envolvidas na comunicação boca a boca pode dar maior credibilidade à informação. As informações transmitidas entre grupos de referência (amigos, colegas, familiares etc.) tendem a apresentar maior credibilidade do que as informações disponibilizadas pelas próprias empresas ofertantes de produtos e serviços. Já uma comunicação formal advinda de uma propaganda empresarial tradicional é baseada em uma apresentação positiva de seus produtos e serviços. Logo, a propaganda tradicional pode vir a gerar um processo de "imunização" ou desconfiança na mente do consumidor (GOLDEMBERG et al, 2001; WIRTZ e CHEW, 2002; MASON, 2008), já que as informações apresentadas pela empresa podem não ser verdadeiras e confiáveis.

Consumidores são influenciados pela comunicação boca a boca devido à sua participação e integração em grupos de referência e ao seu desejo pela redução do risco percebido em suas decisões de consumo. É possível estabelecer, assim, dois constructos centrais na comunicação boca a boca dos consumidores. O primeiro é que a credibilidade da comunicação está atrelada a questões como legitimidade e confiança dos agentes atuantes na comunicação. O segundo é que a rede pela qual a comunicação se estabelece (considerando-se as conexões existentes entre os agentes) caracteriza-se como uma rede social dinâmica (SHETH e PARVATIYAR, 2000). Dessa forma, pode-se afirmar que, quando a credibilidade entre os agentes e as conexões entre os membros da rede de relacionamentos é considerada alta, a comunicação boca a boca tende a ter maior influência sobre as ações e opiniões dos consumidores. Assim, os consumidores consideram as opiniões de seus grupos de referências no momento dos seus processos decisórios de consumo a fim de diminuir a incerteza e o risco percebido.

Apesar de ser considerado inicialmente como um fenômeno espontâneo, a comunicação boca a boca pode ser fomentada por meio de ações específicas de empresas, como disponibilização de mecanismos e ferramentas virtuais e promoções. Segundo a WOMMA (Word of Mouth Marketing Association) (2010), o investimento mundial em iniciativas de comunicação boca a boca estimulada (Word of Mouth Marketing), tanto por meio de iniciativas pessoais em ambiente físico quanto em ambiente virtual, foi, em 2008, de cerca de US\$1,58 bilhões e tem expectativas de crescimento de aproximadamente $100 \%$ até 2013 . Acredita-se que o crescimento e a velocidade da conexão entre as pessoas, fomentada principalmente por tecnologias virtuais, farão da comunicação boca a boca estimulada uma estratégia primordial dentro da área de marketing (MOORE, 2009).

A comunicação boca a boca apresenta um forte impacto na formação de expectativas dos consumidores, além de ser determinante no comportamento de compra futura (GRÖNROOS, 2004; RICHINS, 1983). Por se tratar de uma comunicação informal estabelecida entre consumidores, a comunicação boca a boca pode ser positiva ou negativa. O boca a boca negativo apresenta um impacto prejudicial e negativo na imagem da empresa opinada e avaliada, deixando-a em uma posição de maior dificuldade para atrair novos clientes. As experiências negativas vivenciadas pelos consumidores são motivadores fortes de boca a boca negativo, influenciando fortemente atitudes e comportamentos das pessoas que recebem esse tipo de opinião e informação (HAWKINS et al. 2007). A comunicação boca a boca é mais poderosa do que as informações de fontes comerciais, já que é percebida pelo sujeito como mais imparcial (RICHINS, 1983). Dessa forma, a comunicação boca a boca negativa tem maior força do que a positiva devido a questões emocionais que circundam a insatisfação, tais como decepção, frustração e raiva. Assim, consumidores insatisfeitos apresentam necessidades mais urgentes de divulgar suas insatisfações e frustrações, o que leva a comunicação boca a boca negativa a se tornar mais frequente e mais impactante (BLACKWELL, ENGEL e MINIARD, 2005). 
Apesar de a comunicação boca a boca ser estudada tradicionalmente sob a ótica da comunicação que ocorre por meio de interação pessoal e presencial entre indivíduos (BANSAL e VOYER, 2000; BROWN e REINGEN, 1987), observa-se uma ascensão da comunicação boca a boca em ambientes de compra virtuais (DELLAROCAS, 2003). Assim, os consumidores estão sujeitos à comunicação boca a boca eletrônica (e-WOM) por meio de web sites, blogs, salas de bate-papo, e-mail e redes sociais virtuais (HENNIG-THURAU et al., 2004; GOLDSMITH e HOROWITZ, 2006).

\section{REDES SOCIAIS E REDES SOCIAIS VIRTUAIS}

O conceito de redes sociais pode ser definido como um conjunto de dois elementos. O primeiro são os atores que representam as pessoas, as instituições ou os grupos, que podem ser considerados como os elementos sociáveis. Já o segundo elemento são as conexões, ou seja, as ligações existentes entre os atores da rede (WASSERMAN e FAUST, 1994; DEGENNE e FORSÉ, 1999). As conexões entre dois atores de uma rede social são chamadas de laço social, que é denominado como aquilo que estabelece uma ligação entre um par de atores (WASSERMAN e FAUST, 1994).

Um laço social é formado por relações sociais, as quais são constituídas por interações sociais. Essas interações são ações baseadas em aspectos comunicativos entre os indivíduos e seus contatos sociais (WATZLAVICK, BEAVIN e JACKSON, 2000). Ocorrendo de forma recorrente, essas interações se tornam relações sociais por meio da construção de laços relacionais. Outra forma de laço social são os laços associativos, caracterizados pelo sentimento de participação e pertencimento a um grupo ou instituição, independente das relações sociais observadas nos laços relacionais (BREIGER, 1974).

Laços sociais também podem ser avaliados em termos de força, de modo que a força dos laços é a combinação, possivelmente linear, do tempo, da intensidade emocional, da intimidade e da reciprocidade existente entre os atores que formam o laço (GRANOVETTER, 1973). Laços fortes são, portanto, caracterizados pela intimidade, proximidade e intencionalidade dos atores para criar e manter conexões. Em contrapartida, os laços fracos são caracterizados por relações esparsas, com pouca proximidade e intimidade entre os atores. As trocas sociais estão mais presentes nos laços fortes (WELLMAN, 1997). Portanto, a força dos laços impacta positivamente as trocas sociais em termos de comunicação e influência.

A pesquisa acerca das redes sociais assume um patamar importante nos estudos sociais, já que se sabe que os indivíduos não podem ser estudados independentemente de suas relações com outros indivíduos e de suas estruturas de afiliação (DEGENNE e FORSÉ, 1999). Seguindo essa lógica, o estudo da inserção dos indivíduos em redes sociais engloba o fenômeno das redes sociais virtuais e seus impactos nos comportamentos individuais.

Redes sociais virtuais são redes de laços sociais conduzidas por meio da internet. Dessa forma, as redes sociais virtuais funcionam por meio de interações sociais em ambiente virtual que conectam pessoas e proporcionam comunicação, forjando laços sociais (RECUERO, 2004). As redes sociais virtuais, em sua grande maioria, são caracterizadas por laços sociais fracos, diversificados e possivelmente especializados (CASTELLS, 1999). Em contrapartida, as redes sociais virtuais não estão atreladas a limites geográficos, podendo ser construídas de forma mais dispersa e ampla. Com isso, o ambiente virtual encoraja relacionamentos independentemente da posição geográfica dos atores e de condições temporais para que as interações ocorram (LÉVY, 1999).

As ferramentas e funcionalidades das redes sociais constituídas por meio da internet (exemplo: Orkut, Facebook, MySpace e Twitter) são em geral gratuitas e de fácil utilização. Além 
disso, oferecem espaço virtual para a postagem de conteúdos opinativos e manutenção de contatos e interações entre atores independentemente do tempo e posição geográfica. Apesar de ser considerada uma ferramenta que proporciona condições de ampliar as redes sociais tradicionais por meio de laços fracos com atores dispersos, a quantidade de interações possíveis pode vir a fortalecer as conexões já existentes em detrimento das limitações temporais e locacionais das redes sociais tradicionais. Dentre as redes sociais mais acessadas no mundo, estão o Facebook (cerca de 1,2 bilhões de acessos por mês), o MySpace (cerca de 810 milhões de acessos por mês) e o Twitter (cerca de 54 milhões de acessos por mês) (COMPETE, 2009). No Brasil, as redes sociais virtuais mais acessadas, em agosto de 2010, foram o Orkut (cerca de 29,4 milhões de acessos), o Windows Live Profile (cerca de 12,5 milhões de acessos) e o Facebook (cerca de 8,9 milhões de acessos) (COMSCORE, 2010).

Estudos que abordam a influência do acesso e de contatos em redes sociais virtuais nos comportamentos de consumidores ainda são poucos e superficiais. Logicamente, a investigação desse tema carece de estudos aprofundados e, possivelmente, de estudos etnográficos. No entanto, algumas evidências interessantes são encontradas. Em estudo realizado por Xiao, Li, Cão e Tang (2012), tendo como proposta entender a troca de conhecimentos em comunidade virtual a partir de uma perspectiva social, os resultados sugeriram que os atributos sociais on-line dos usuários da internet são importantes facilitadores de intercâmbio de conhecimento em comunidades virtuais. Logo, os laços sociais virtuais facilitam a troca de conhecimentos e, possivelmente, opinições sobre produtos e serviços. Já outro estudo de levantamento evidenciou que membros participantes de comunidades virtuais são mais propensos a explorar outras áreas comerciais virtuais fora de seu padrão de consumo, o que os tornam mais rentáveis para sites de vendas on-line (OLBRICH e HOLSING, 2011). Apesar de poucos estudos serem focados nesse tema investigativo, cada vez mais os consumidores interagem por meio da internet para partilhar seus conhecimentos, experiências e opiniões. Logo, o boca a boca eletrônico tornou-se uma força de mercado significativa que influencia a tomada de decisão do consumidor (DE VALCK, VAN BRUGGEN e WIERENGA, 2009). No Brasil, não foram encontrados estudos consubstanciados que abordem o tema, o que representa uma lacuna acadêmica importante.

\section{METODOLOGIA}

Este estudo se configura como uma pesquisa de levantamento (survey) (BABBIE, 1999) descritiva quanto aos seus objetivos. Os participantes foram estudantes universitários de uma Instituição de Ensino Superior (IES) particular localizada na cidade de Santa Maria, Rio Grande do Sul. Com relação à natureza, essa pesquisa se classifica como quantitativa. Detalhes a respeito da condução deste estudo são apresentados nas próximas subseções.

\subsection{Elaboração do instrumento de coleta de dados}

O instrumento de coleta de dados utilizado foi um questionário composto por 11 questões fechadas, utilizadas para determinar o perfil comportamental dos respondentes de acordo com os objetivos deste estudo. Essas questões foram adaptadas de escalas originais já previamente definidas e testadas por outros pesquisadores para avaliar a Suscetibilidade de Influência Interpessoal (MASCARENHAS e HIGBY, 1993), a Liderança de Opinião (WELLS e TIGERT, 1971) e o Uso de Informações de Anúncios (LUMPKIN e DARDEN, 1982). As mesmas foram adaptadas para mensurar: (1) a Suscetibilidade de Influência Interpessoal em Ambiente Virtual; (2) a Liderança 
de Opinião em Ambiente Virtual; e (3) a Utilização de Informações Postadas por Contatos Virtuais. Essas escalas podem ser vistas no quadro 1, com suas respectivas variáveis.

\begin{tabular}{|c|c|c|}
\hline DIMENSÃO & ITENS ADAPTADOS & $\begin{array}{l}\text { AUTORES } \\
\text { ORIGINAIS }\end{array}$ \\
\hline $\begin{array}{l}\text { Influência Interpessoal } \\
\text { em Ambiente Virtual. } \\
\text { Escala Original: Interper- } \\
\text { sonal Influence Susceptibi- } \\
\text { lity (Media-Normative) }\end{array}$ & $\begin{array}{l}\text { V1- Eu compro apenas produtos/marcas que são comen- } \\
\text { tadas positivamente na internet (fóruns, blogs e redes } \\
\text { sociais virtuais). } \\
\text { V2- Postagens de outros usuários na internet determi- } \\
\text { nam minha lealdade a marcas. } \\
\text { V3- Eu continuo comprando as mesmas marcas enquan- } \\
\text { to estas são avaliadas positivamente em fóruns, blogs e } \\
\text { redes sociais virtuais. }\end{array}$ & $\begin{array}{l}\text { Mascarenhas e } \\
\text { Higby (1993). }\end{array}$ \\
\hline $\begin{array}{l}\text { Influência Interpessoal } \\
\text { em Ambiente Virtual. } \\
\text { Escala Original: Interper- } \\
\text { sonal Influence Susceptibi- } \\
\text { lity (Parent-Informative.) }\end{array}$ & $\begin{array}{l}\text { V4- Eu nunca compro nenhum novo produto a menos } \\
\text { que já tenha buscado opiniões na internet sobre o mes- } \\
\text { mo. } \\
\text { V5- Quando eu não entendo os preços e as qualidades } \\
\text { de produtos, eu consulto opiniões na internet. } \\
\text { V6- Eu frequentemente busco discutir sobre meus pla- } \\
\text { nos de compra com outros usuários na internet. }\end{array}$ & $\begin{array}{l}\text { Mascarenhas e } \\
\text { Higby (1993). }\end{array}$ \\
\hline $\begin{array}{l}\text { Liderança de Opinião em } \\
\text { Ambiente Virtual. } \\
\text { Escala Original: Opinion } \\
\text { Leadership (Product) }\end{array}$ & $\begin{array}{l}\text { V7- Meus contatos e amigos virtuais frequentemente } \\
\text { me procuram para perguntar minhas opiniões. } \\
\text { V8- Eu, às vezes, influencio o que meus amigos e conta- } \\
\text { tos virtuais compram. } \\
\text { V9- Meus contatos e amigos virtuais vêm mais a mim do } \\
\text { que eu até eles para buscar opiniões relativas a produtos } \\
\text { e marcas. }\end{array}$ & $\begin{array}{l}\text { Wells e Tigert } \\
\text { (1971). }\end{array}$ \\
\hline $\begin{array}{l}\text { Utilização de Informações } \\
\text { Postadas por Contatos } \\
\text { Virtuais. } \\
\text { Escala Original: Ad Infor- } \\
\text { mation Usage. }\end{array}$ & $\begin{array}{l}\text { V10- Eu frequentemente leio opiniões e comentários de } \\
\text { outras pessoas na internet sobre produtos, serviços e } \\
\text { marcas. } \\
\text { V11- Comentários e opiniões de usuários da internet são } \\
\text { a fonte de informação que eu uso quando estou queren- } \\
\text { do comprar algo. }\end{array}$ & $\begin{array}{l}\text { Lumpkin e Dar- } \\
\text { den (1982). }\end{array}$ \\
\hline
\end{tabular}

Quadro 1 - Escalas utilizadas nesta pesquisa.

De acordo com as sugestões propostas por Hair et al. (2005) e Garver e Mentzer (1999), a avaliação e validação do instrumento de coleta e das escalas de mensuração para os constructos mencionados anteriormente foram realizadas por meio do cálculo do Alfa de Cronbach (CRONBACH, 1951), da confiabilidade composta e da variância extraída (AVE). Esses dois últimos são efetuados por meio da soma das cargas das variâncias padronizadas e dos erros de mensuração das variáveis. Os 11 itens do instrumento foram mensurados com uma escala Likert de concordância de 5 pontos.

Tendo como objetivo caracterizar o perfil dos respondentes, foram também incluídas no questionário questões relativas ao sexo, à idade, à escolaridade, ao estado civil e à renda familiar. Também foram elaboradas questões para identificar as mídias sociais em que os participantes do estudo tinham cadastro e que utilizavam com maior frequência.

\subsection{Coleta de dados}

Este estudo foi conduzido por meio de um survey eletrônico (BABBIE, 1999; HAIR, et. al, 2005). Elaboraram-se questionários estruturados, que foram enviados por e-mail para uma amostra de estudantes universitários, juntamente com instruções detalhadas para o seu correto preenchimento.

O tipo de amostragem utilizado caracteriza-se como não probabilística. Na amostragem não probabilística existe uma confiança no julgamento pessoal do pesquisador, pois ele decide os 
elementos a serem incluídos na amostra (MALHOTRA, 2001). Este estudo foi realizado com uma amostra não probabilística por conveniência, formada por 200 estudantes universitários de uma IES particular localizada em Santa Maria, RS. A população de estudantes universitários nessa IES na ocasião da pesquisa era de cerca de 7.500 alunos. Logo, a margem de erro para tal população é de $7 \%$, o que é considerado positivo.

É aconselhado que, para cada item do questionário baseado em uma escala, deve-se ter em torno de cinco respondentes (HAIR et. al, 2005). Assim, o tamanho da amostra deste estudo é suficiente, já que a escala inicial adotada é composta por 11 itens que mensuram as variáveis chaves do estudo. A coleta de dados ocorreu nos meses de outubro e novembro de 2010 mediante a aplicação de um questionário estruturado no formsite sobre as redes sociais virtuais e as fontes de informação utilizadas para decisão de compra, enviado por e-mail à população pesquisada.

Os dados obtidos foram tabulados com ajuda do formsite e do software SPSS. Os dados foram tratados com base em técnicas estatísticas descritivas univariadas e multivariadas. Dentre as técnicas multivariadas, adotaram-se a Análise de Componentes Principais e com a Análise Fatorial Exploratória.

\subsection{Análise de dados}

Os dados obtidos foram analisados inicialmente por meio de técnicas de estatística descritiva. Foram observados os valores de média (como medida de tendência central) e desvio padrão (como medida de dispersão). Após essa etapa, foram aplicadas técnicas de análise fatorial exploratória (AFE) nos 11 indicadores provenientes das escalas já citadas anteriormente. Por meio da AFE, foram obtidos dois componentes principais, adotando-se o critério de avaliação de Kaiser (com autovalores $>1$ ). Os valores das cargas fatoriais foram rotacionados ortogonalmente pelo método Varimax. Além disso, foram observados os valores do alfa de Cronbach, da confiabilidade composta e da variância média extraída (AVE). Esses dois últimos valores são calculados a partir das cargas fatoriais e dos erros padrões (HAIR et. al, 2005).

Em seguida, foram conduzidas duas análises de regressão múltipla, tendo como variáveis dependentes as variáveis latentes oriundas da AFE. As variáveis independentes dos modelos foram gênero, idade, renda familiar e tempo de uso semanal de internet dos estudantes universitários pesquisados. Salienta-se que, com exceção do gênero (variável dummy), as demais variáveis independentes foram avaliadas de forma escalar. Também foi observada a correlação de Pearson existente entre as variáveis latentes que avaliam o comportamento dos estudantes pesquisados. Para fins de análise estatística dos dados coletados, contou-se com o auxílio do software SPSS for Windows 15.0.

\section{ANÁLISE DOS RESULTADOS}

Nesta seção, são apresentadas as informações referentes ao perfil dos respondentes. Em seguida, são apresentados os dados quantitativos relativos aos objetivos centrais deste estudo e que caracterizam o comportamento dos respondentes no que tange às ações de busca e disseminação de informações em redes sociais virtuais a respeito de produtos e serviços.

\subsection{Perfil dos respondentes}

Da totalidade dos entrevistados, $59,5 \%$ são do sexo feminino e 40,5\% são do sexo masculino. A faixa etária predominante é de 19 a 25 anos (74,5\% dos entrevistados). Além disso, 
$90 \%$ dos entrevistados são solteiros. A maioria dos respondentes $(84,5 \%)$ está cursando o ensino superior e outros $15,5 \%$ já possuem algum curso superior completo, apesar de estarem cursando alguma graduação na IES pesquisada. Já com relação à renda líquida familiar, observou-se que $32,5 \%$ dos entrevistados possuem uma renda líquida familiar variando de 5 a 10 salários mínimos, $24,5 \%$ possuem renda líquida familiar entre 3 e 5 salários mínimos e outros $22 \%$ possuem renda superior a 10 salários mínimos.

Uma constatação interessante foi a de que $76,5 \%$ dos entrevistados afirmam utilizar a internet como o principal meio para pesquisar sobre produtos. Com relação ao cadastro em mídias sociais, o Orkut apareceu em primeiro lugar, com 95,5\% de entrevistados cadastrados, seguido pelo Twitter, com $42 \%$, e pelo Facebook, com $35 \%$. Quanto às mídias que os usuários mais acessavam na ocasião da coleta de dados, novamente os entrevistados apontaram o Orkut em primeiro lugar, com $88,5 \%$ da preferência, seguido pelo Twitter, com $25 \%$. Assim, pode-se afirmar que o Orkut e o Twitter eram, no momento da realização da coleta de dados da presente pesquisa, os serviços mais conhecidos e utilizados pelos internautas entrevistados. No entanto, devido à dinamicidade do meio virtual, esses dados podem não mais representar a realidade.

Dentre as motivações para o uso das redes sociais virtuais, estão: possibilidade de contato com os amigos (97,5\%), fonte de pesquisa sobre produtos ou serviços $(66 \%)$ e fonte de obtenção de informações sobre lazer e entretenimento (58\%). Dessa forma, destaca-se o considerável percentual de resultados que afirma a utilização das redes sociais como fonte de pesquisa sobre produtos/serviços; porém, pode-se dizer que o público pesquisado ainda não desenvolveu fortemente o hábito de expor nessas mídias sua própria opinião quanto a produtos/serviços.

Com relação às atividades já realizadas na internet, pela amostra pesquisada, verifica-se que $78 \%$ dos respondentes afirmam já ter realizado compras por meio da internet. Quase $75 \%$ da amostra entrevistada afirma ler ou ao menos já ter lido blogs, e quase $62 \%$ já deixou comentários pessoais em blogs, fóruns ou comunidades.

Quanto ao percentual de entrevistados que já sofreu influência das redes sociais ao comprar produtos/serviços/marcas, observa-se que a maioria da amostra pesquisada $(82,5 \%)$ revela já ter tido suas compras influenciadas por informações contidas em redes sociais.

\subsection{Resultados analíticos do estudo}

Ao analisar as médias das respostas dos entrevistados, percebe-se que o grau de concordância é maior que 3 para as questões que se referem às redes sociais influenciando o comportamento de compra dos entrevistados. Com isso, os resultados sugerem que os pesquisados concordam que consultam opiniões na internet quando não entendem preço e qualidades de produtos (média=4,045). Além disso, os respondentes concordam que a fonte de informação que mais usam ao decidir comprar algo são comentários e opiniões de usuários da internet (média=3,360). Por outro lado, as evidências sugerem que os estudantes pesquisados tendem a buscar mais informações do que a disseminá-las a seus contatos, o que pode ser observado nos valores de média aritmética obtidos para as questões 7,8 e 9 .

Dando continuidade à análise dos dados, os procedimentos multivariados de análise estatística propõem a definição de alguns índices que determinam a confiabilidade e validade da escala adotada. O Alfa de Cronbach (CRONBACH, 1951), que serve para mensurar o grau de confiabilidade interno do instrumento de coleta de dados da pesquisa, é de 0,837 , ou seja, a escala mede aquilo que se propõe a mensurar de modo altamente satisfatório. Porém, esse valor não leva em conta o item 6, devido a uma divergência na análise fatorial exploratória (os resultados 
são apresentados a seguir). Já a verificação da adequação da amostra foi avaliada pelo Teste de esfericidade de Bartlett e pelo índice Kaiser-Meyer-Olkin (KMO), os quais se apresentaram altamente positivos $\left(X^{2}=795,81, p<0,001 ; K M O=0,814\right)$.

Foi realizada em seguida uma Análise Fatorial Exploratória com os 11 itens originais da escala utilizada. A partir de uma redução para 2 componentes, em que foi empregado o critério de Kaiser (autovalor $>1$ ), as 11 variáveis originais geraram duas novas variáveis latentes: propensão a ser influenciado e capacidade de influenciar. As cargas fatoriais obtidas nos componentes foram rotacionadas pelo método Varimax, e o item 6 foi excluído em função do seu valor de carga fatorial menor do que 0,5 em ambos os componentes. No quadro a seguir, são apresentados os valores das cargas fatoriais significativas de cada componente, assim como os valores de Eigenvalue, da Variância explicada e do Alfa de Cronbach.

Tabela 1 - Análise Fatorial Exploratória dos resultados.

\begin{tabular}{|c|c|c|c|c|c|c|c|}
\hline Dimensão & Itens & $\begin{array}{c}\text { Carga } \\
\text { fatorial }\end{array}$ & Média & $\begin{array}{l}\text { Desvio } \\
\text { Padrão }\end{array}$ & $\begin{array}{l}\text { Alfa de } \\
\text { Cronbach }\end{array}$ & $\begin{array}{c}\text { Confiabilidade } \\
\text { Composta }\end{array}$ & AVE \\
\hline \multirow{7}{*}{$\begin{array}{l}\text { Propensão a ser } \\
\text { influenciado }\end{array}$} & V.1 & $0,770 *$ & 3,505 & 1,129 & \multirow{7}{*}{0,831} & \multirow{7}{*}{0,861} & \multirow{7}{*}{0,473} \\
\hline & V.2 & $0,781 *$ & 3,185 & 1,125 & & & \\
\hline & V.3 & $0,714 *$ & 3,620 & 1,025 & & & \\
\hline & V.4 & $0,563 *$ & 3,165 & 1,329 & & & \\
\hline & V.5 & $0,561 *$ & 4,045 & 1,038 & & & \\
\hline & V.10 & $0,642 *$ & 3,780 & 1,103 & & & \\
\hline & V.11 & $0,743 *$ & 3,360 & 1,215 & & & \\
\hline \multirow{3}{*}{$\begin{array}{l}\text { Capacidade de } \\
\text { influenciar os outros }\end{array}$} & V.7 & $0,874 *$ & 2,735 & 1,229 & \multirow{3}{*}{0,856} & \multirow{3}{*}{0,902} & \multirow{3}{*}{0,754} \\
\hline & V.8 & $0,859 *$ & 2,855 & 1,249 & & & \\
\hline & V.9 & $0,872 *$ & 2,650 & 1,128 & & & \\
\hline
\end{tabular}

Nota: ${ }^{*} p<0,001$

O componente 1 , devido à natureza das variáveis que o compõem, foi chamado de "propensão de ser influenciado". Já o componente 2 foi considerado como a "capacidade de influenciar os outros". A variância total explicada por esses dois fatores é de $58,91 \%$, índice considerado suficiente em ciências sociais e altamente positivo tendo em vista a utilização de apenas dois componentes principais. Assim, a escala adaptada para avaliar o comportamento dos usuários universitários, no que tange à troca de informações e opiniões em redes sociais virtuais, é considerada válida. A seguir, são apresentados os resultados das análises de regressão múltipla conduzidas.

Tabela 2 - Resultados da Análise de Regressão Múltipla

\begin{tabular}{ccc}
\hline & Propensão a ser influenciado & Capacidade de influenciar os outros \\
\hline Constante & $13,005(p=0,486)$ & $27,248(p=0,135)$ \\
\hline Tempo $^{b}$ & $\beta=-0,088(p=0,032) *$ & $\beta=0,047(p=0,234)$ \\
Gênero $^{a}$ & $\beta=-0,119(p=0,410)$ & $\beta=-0,510(p<0,001) * * *$ \\
Idade $^{b}$ & $\beta=0,101(p=0,266)$ & $\beta=0,094(p=0,287)$ \\
Renda Familiar $^{b}$ & $\beta=-0,021(p=0,723)$ & $\beta=0,098(p=0,093)$ \\
\hline$R$ & 0,1823 & 0,2927 \\
$R^{2}$ & 0,0332 & 0,0856 \\
$R^{2}$ ajustado & $0,01342(p=0,157)$ & $0,0669(p=0,001) * *$ \\
\hline Nota: $^{*} p<0.05 ;{ }^{* *} p<0.01 ;{ }^{* * *} p<0.001 ;{ }^{a}$ variável dummy $\left(0=\right.$ masculino; $1=$ feminino); ${ }^{b}$ variável escalar.
\end{tabular}


O primeiro modelo de regressão, que tem a variável "propensão a ser influenciado", não apresentou significância estatística $(p=0,157)$. Entretanto, observou-se que o tempo de acesso semanal à internet afeta positivamente e significativamente a propensão dos estudantes universitários a serem influenciados por seus contatos virtuais $(p=0,032)$. Já o segundo modelo, que tem a variável "capacidade de influenciar os outros", apresentou significância estatística $(p=0,001)$. Dentre as variáveis independentes do modelo, apenas o gênero apresentou significância estatística. Os valores sugerem que os homens apresentam capacidade significativamente maior de influenciar outras pessoas do que as mulheres $(p<0,001)$. Apesar de os resultados não serem significativos para algumas variáveis, considera-se que essas averiguações estatisticamente não significativas também sejam pertinentes ao conhecimento científico, uma vez que evidenciam a não significância de tais variáveis.

Outro ponto observado foi a correlação existente entre as duas dimensões abordadas Constatou-se que existe uma correlação positiva e significativa entre a propensão de ser influenciado e a capacidade de influenciar outras pessoas ( $r h o=0,356 ; p<0,001$ ). Esse resultado sugere que quanto mais propensas as pessoas estiverem a serem influenciadas, maior a capacidade delas de influenciar outras pessoas. Assim, a predisposição e abertura das pessoas para opiniões, sugestões e críticas relacionadas a produtos está interligada com a capacidade de influenciar os comportamentos de consumo de outras pessoas.

\section{DISCUSSÕES FINAIS}

A tecnologia digital e a internet permitiram a criação de uma forma muito eficaz de marketing que se baseia na colaboração e no compartilhamento de informações entre os usuários (ELLWOOD, 2004). Além de constituir um meio de facilitar a interação e o relacionamento entre as pessoas, as redes sociais virtuais são utilizadas como uma fonte de informação autêntica na medida em que seus usuários, além de compartilhar seus dados pessoais, dividem suas opiniões sobre diversos assuntos, tais como sobre produtos ou serviços.

Considera-se que o objetivo geral do presente estudo, que foi analisar a postura de estudantes universitários com relação à adoção do ambiente virtual e de redes sociais virtuais no processo decisório de consumo, foi atendido. Com relação ao perfil dos entrevistados, pode-se dizer que são estudantes universitários de 19 a 25 anos, com renda líquida familiar variando de 5 a 10 salários mínimos, que afirmam utilizar a internet como o principal meio para pesquisar sobre produtos ou serviços. O Orkut e o Twitter eram, na ocasião da pesquisa, os serviços mais conhecidos e utilizados pelos internautas entrevistados, com o intuito de manter contato com os amigos, pesquisar sobre produtos e serviços e obter informações sobre lazer e entretenimento. Entretanto, esses dados podem não mais representar a realidade devido ao dinamismo do meio virtual. Dentre as atividades já realizadas por esses usuários na internet, estão compras, leitura de blogs e postagem de comentários pessoais nas redes. Além disso, a maioria dos pesquisados revelaram já ter tido suas compras influenciadas por informações contidas em redes sociais.

Uma das grandes contribuições deste estudo é a utilização de uma escala adaptada para mensurar e avaliar o nível de propensão de influenciar e ser influenciado pelos contatos das redes sociais virtuais. Por meio de escalas tradicionais, foram criados dois constructos analíticos - a propensão a ser influenciado e a capacidade de influenciar os outros em ambiente virtual -, que apresentaram valores satisfatórios de confiabilidade interna, confiabilidade composta e variância extraída. Dessa forma, esses constructos podem vir a ser adotados em outros estudos, gerando relações interessantes com outras variáveis a respeito do comportamento de usuários de redes sociais virtuais. 
Uma evidência interessante é a constatação de que os estudantes pesquisados consideram-se mais propensos a serem influenciados do que capazes de influenciar os outros em ambientes virtuais. Outro resultado relevante relatado neste estudo é a identificação de diferença significativa entre homens e mulheres para a variável latente "capacidade de influenciar os outros". Isso sugere que os homens apresentam uma maior capacidade de influenciar as opiniões e os comportamentos de seus contatos de redes sociais. Em contrapartida, a propensão de ser influenciado não apresentou diferença significativa entre os gêneros. Também foi observado que o tempo de acesso à internet afeta positivamente e significativamente a propensão dos estudantes universitários pesquisados para serem influenciados por seus contatos virtuais. A renda familiar e a idade dos usuários não apresentaram impacto significativo nas variáveis que avaliam o comportamento de influência dos indivíduos (propensão a ser influenciado e a capacidade de influenciar os outros). Essas evidências podem ser justificadas pelas características da amostra. Os estudantes de uma Instituição de Ensino Superior (IES) privada podem apresentar rendas familiares e idades pouco heterogêneas, o que prejudica as inferências do estudo.

Como limitação importante que não pode ser ignorada ressalta-se o fato de a amostra ter sido por adesão e com a participação de estudantes universitários de apenas uma IES localizada em um município específico. Isso impede a generalização dos resultados obtidos. Além disso, vale lembrar que, pelo fato de a pesquisa ter sido conduzida em 2010, algumas mudanças com relação aos resultados aqui obtidos podem não representar a atualidade. Sugerem-se estudos futuros que relacionem a adoção de redes sociais virtuais e os comportamentos individuais e coletivos de consumo.

\section{REFERENCIAS}

$B A B B I E, E$. Métodos de pesquisa de survey. Belo Horizonte: Ed. UFMG, 1999.

BANSAL, H. S.; VOYER, P. A. Word-of-mouth processes within a services purchase decision context. Journal of Service Research. v. 3, n. 2, p.166-177, 2000.

BLACKWELL, R. D.; MINIARD, P. W.; ENGEL, J. F. Comportamento do consumidor. 9. ed. São Paulo, SP: Pioneira Thomson Learning, 2005.

BREIGER, R. The Duality of Persons and Groups. Social Forces. v. 53, n. 2, p. 181- 190, 1974.

BROWN, J. J.; REINGEN, P. H. Social ties and word-of-mouth referral behavior. Journal of Consumer Research. v. 14, n. 3, p. :350-362, 1987.

CASTELLS, Manuel. A sociedade em rede. 2. ed. São Paulo: Paz e Terra, 1999.

COMPETE. Social Networks: Facebook Takes Over
Top Spot, Twitter Climbs (2009). Disponível em <http://blog.compete.com/2009/02/09/facebookmyspace-twitter-social-network/>. Acesso em 02 de Nov. de 2010.

COMSCORE. Orkut Continua Liderando o Mercado de Redes Sociais no Brasil, e a Audiência do Facebook Quintuplica (2010). Disponível em: <http://www.comscore.com/ por/layout/set/popup/Press_Events/Press_ Releases/2010/10/Orkut_Continues_to_ Lead_Brazil_s_Social_Networking_Market_ Facebook_Audience_Grows_Fivefold $>$. Acesso em 02 de Nov. de 2010.

CRONBACH L. J. Coefficient alpha and the internal structure of tests. Psychometrika. v. 16, p. 297-334, 1951.

DAMBRÓS, Juliana. Marca nas Redes Sociais Virtuais: Uma proposta de Gestão Colaborativa através do relacionamento com o consumidor. Trabalho de conclusão de curso de 
Comunicação Social - Habilitaçao Publicidade e Propaganda. Universidade Regional de Blumenau. Blumenau. 2007. Disponível em: < http://migre.me/1vw7x >. Acesso em: 15 mai. 2010.

DE VALCK, K.; VAN BRUGGEN, G. H.; WIERENGA, B. Virtual communities: A marketing perspective. Decision Support Systems, v. 47, n. 3, p. 185-203, 2009.

DEGENNE, Alain e FORSÉ, Michel. Introducing Social Networks. London: Sage, 1999.

DELLAROCAS, C. The digitization of wordof-mouth: promise and challenges of online feedback mechanisms. Management Science. v. 49, n. 10, p. 1407-1424, 2003.

ELLWOOD, lain. 0 livro essencial das marcas: Tudo o que você precisa saber, em mais de 100 técnicas para aumentar o valor das marcas. São Paulo: Clio Editora, 2004.

FERRARI, Bruno. Onde os brasileiros se encontram. Revista Época. São Paulo, n. 628, p. 84-85, 31 maio 2010.

GARVER, M. S.; MENTZER, J. T. Logistics research methods: employing structural equation modeling to test for construct validity. Journal of Business Logistics, v. 20, n. 1, p. 3357, 1999.

GOLDEMBERG, J.; LIBAI, B.; MULLER, E. Talk the network: a complex system look at the underlying processes of word-of-mouth. Marketing Letters, v. 12, n. 3, p 211-223, 2001.

GOLDSMITH, R. E.; HOROWITZ, D. Measuring motivations for online opinion seeking. Journal of Interactive Advertising. v. 6, n. 2, p. 1-16, 2006.

GRANOVETTER, M.S. The strength of weak ties. American Journal of Sociology, v. 78, n. 6, p.13601380, 1973.

HAIR, JR., Joseph F.; ANDERSON, Rolph E.; TATHAM, Ronald L.; BLACK, William C. Análise multivariada de dados. 5. ed. Porto Alegre: Bookman, 2005.

HAWKINS, D. I.; MOTHERSBAUGH, D. L.; BEST, R. J. Consumer Behavior: Building Marketing Strategy. 10. ed. New York: McGraw-Hill, 2007.

HENNIG-THURAU, T.; GWINNER, K. P.; WALSH, G.; GREMLER, D. D. Electronic word-ofmouth via consumer opinion platforms: what motivates consumers to articulate themselves on the internet? Journal of Interact Marketing. v. 18, n. 1, p. 38-52, 2004.

IBGE - Instituto Brasileiro de Geografia e Estatística. Pesquisa Nacional por Amostra de Domicílios (2005 e 2009). Disponível em: <http://www.ibge.gov.br>. Acesso em: 12 jun, 2012.

JEFFREY GROUP. Empresas e consumidores nas mídias sociais: A visão dos heavy users sobre a comunicação e o relacionamento com empresas e marcas na rede. Disponível em: <www.job360.com.br/nos/empresas consumidores_midiassociais.pdf $>$. Acesso em 13 jun, 2012.

KIMMEL, A.J. Rumors and rumor control. Lawrence Erlbaum: New Jersey, 2003.

LÉVY, Pierre. Cibercultura. São Paulo: Ed. 34, 1999.

LUMPKIN, James R.; DARDEN, William R. Relating Television Preference Viewing to Shopping Orientations, Lifestyles, and Demographics. Journal of Advertising. v. 11, n. 4, p. 56-67, 1982.

MALHOTRA, Naresh. Pesquisa de marketing: Uma Orientação Aplicada. 3. ed. Porto Alegre: Bookman, 2001.

MASCARENHAS, Oswald A. J.; HIGBY, Mary A. Peer, Parent, and Media Influences in Teen Apparel Shopping. Journal of the Academy of Marketing Science. v. 21, n. 1, p. 53-58, 1993.

MASON, R.B. Word of mouth as a promotional 
tool for turbulent markets. Journal of Marketing Communications. v. 14, n. 3, p. 207-224, 2008.

MOORE, J. Word of Mouth Forecast 20092013: Spending, Trends \& Analysis, 2009. Disponível em: <http://brandautopsy.typepad. com/brandautopsy/2009/07/pq-mediawomforecast-report.html>. Acesso em: 014.09.10.

OLBRICH, R.; HOLSING, C. Modeling Consumer Purchasing Behavior in Social Shopping Communities with Clickstream Data. International Journal of Electronic Commerce, v. 16, n. 2, p. 15-40, 2011.

RECUERO, Raquel da Cunha. Teoria das Redes e Redes Sociais na Internet. Trabalho apresentado no XXVII INTERCOM, na PUC/RS em Porto Alegre. Setembro de 2004.

RICHINS, M. L. Negative Word-of-Mouth by Dissatisfied Consumers: A Pilot Study. Journal of Marketing. v. 47, n. 1, p. 68-78, 1983.

SEGALLA Amauri, RIBEIRO Aline, BARIFOUSE Rafael. O poder do consumidor no mundo digital. Época Negócios Ed 8 outubro 2007. Disponível em: <http://epocanegocios.globo. com/Revista/Epocanegocios/0,,EDG794238382-8-1,00-O+PODER+DO+CONSUMIDOR+ NO+MUNDO+DIGITAL.html >. Acesso em: 1 mai. 2010.

SHETH, Jagdish; PARVATIYAR, Atul. Handbook of Relationship Marketing. London: Sage Publications, Inc., 2000.

STANLEY, Chris. A Web 2.0 e o Marketing das redes sociais. HSM Management, 75ed. Jul 2008. Disponível em < http:// br.hsmglobal.com/adjuntos / 14 / documentos/000/073/0000073470.pdf >. Acesso em: 28 mar. 2010.

WASSERMAN, Stanley; FAUST, Katherine. Social Network Analysis: Methods and Applications. Cambridge, UK: Cambridge University Press, 1994.
WATZLAVICK, Paul, BEAVIN, Janet e JACKSON, Don. Pragmática da Comunicação Humana. 11ํ. Ed. São Paulo: Cultrix, 2000.

WELLMAN, Barry. An Electronic Group is Virtually a Social Network. In KIESLER, Sarah (org.) Culture of Internet. Hilsdale, NJ: Lawrence Erlbaum, 1997.

WELLS, William D.; TIGERT, Douglas. Activities, Interests, and Opinions. Journal of Advertising Research. v. 11, n. 4, p. 27-35, 1971.

WIRTZ, J.; CHEW, P. The Effects of Incentives, Deal Proneness, Satisfaction and Tie Strength on Word-Of-Mouth Behavior. International Journal of Industry Management, v. 13, n. 2, p.141-162, 2002.

WOMMA. World of Mouth Marketing Association. Disponível em: <http://womma. org/>. Acesso em 10 de set. de 2010.

XIAO, H. L.; LI, W. F.; CAO, X. B.; TANG, Z. M. The Online Social Networks on Knowledge Exchange: Online Social Identity, Social Tie and Culture Orientation. Journal of Global Information Technology Management, v. 15, n. 2, p. 4-24, 2012. 